\title{
NK/T-cell lymphoma with inverted papilloma: A rare coexistence
}

\author{
Asitava Deb Roy, Isha Preet Tuli, Deepti Joshi
}

Sikkim Manipal Institute of Medical Sciences, Gangtok, Sikkim, India

\section{CASE STUDY}

Please cite this paper as: Deb Roy A, Tuli IP, Joshi D. NK/T cell lymphoma with inverted papilloma: A rare coexistence. AMJ 2014, 7, 8, 318-322. http://doi.org/10.21767/AMJ.2014.2025

Corresponding Author:

Asitava Deb Roy

Ashray, Anandanagar Lane 3,

Adabari, Guwahati 781012,

Assam, India

Email: asitavadr@gmail.com

\section{ABSTRACT}

Natural killer/T-cell (NK/T) lymphomas are an infrequent tumour type of NK and NK-T cells commonly occurring in the upper aero-digestive tract. Most reported cases in the literature are random solitary cases of NK/T-cell lymphoma. A 35-year-old male farmer from Sikkim reported to our institution with NK/T-cell lymphoma (nasal type) with coexistent inverted papilloma of the nose. This case is being reported due to the unique and unusual simultaneous occurrence of these two tumour entities.

\section{Key Words}

NK/T-cell lymphoma, inverted papilloma, nasal, tumour coexistence

\section{Implications for Practice:}

\section{What is known about this subject?}

NK/T-cell lymphoma is a rare tumour, but has been reported in various parts of the world. However, its coexistence with a benign pathology-like inverted papilloma has rarely been reported.

\section{What new information is offered in this case study?}

This case is unusual with respect to its clinical presentation. The patient had two different yet co-existing nasopharyngeal pathologies, one benign and the other malignant. Despite an extensive literature search, no case of simultaneous occurrence of inverted papilloma with T-cell lymphoma could be found.

3. What are the implications for research, policy, or practice?

Although rare, physicians should be careful in considering the presence of two pathologies of different nature and prognosis. Clinically suspicious cases may be subjected to frozen section biopsy during surgery to rule out malignancy. Our report emphasises the need for awareness about such coexisting pathologies and proper communication between clinicians and pathologists.

\section{Background}

Natural killer/T-cell (NK/T) lymphomas represent a group of rare tumours of NK and NK-T cells, frequently seen in Asian, and Central and South American populations. ${ }^{1}$ The nasaltype NK/T-cell lymphoma is the most common and best characterised variant. This tumour shows a predilection for the upper aero-digestive tract, accounting for the historic terms lethal midline granuloma and midline reticulosis. Most reported cases in the literature are random solitary cases of NK/T-cell lymphoma. Its simultaneous occurrence with other aero-digestive tumours has not been described. $^{2,3}$ We present a rare coexistence of NK/T-cell lymphoma (nasal type) with inverted papilloma of the nose in a 35-year-old male farmer from Sikkim.

\section{Case details}

A previously healthy 35-year-old male farmer from South Sikkim, India, presented in December 2012 to the Otorhinolaryngology Department with a one-year history of nasal obstruction and two-month history of blood tinged nasal discharge, which had not resolved despite several courses of antibiotics. The nasal obstruction was of insidious onset, initially right-sided and intermittent, but it gradually progressed to become persistent, bilateral, and complete. The nasal discharge was thin, pale, watery, not foul smelling, and moderate in amount. There was occasional blood tingeing of the discharge, particularly after sneezing. 
He also had foreign body sensation in the throat two months prior to presentation, along with a dull pain while swallowing solid food and a hyponasal voice. He gave no history of any night sweats, fever, cough, hawking, facial pain, frank epistaxis, dyspnea, otalgia, addiction, tuberculosis, or diabetes.

Physical examination revealed a large multi-lobulated, polypoidal, pale mass completely filling the right nasal cavity. On probing it was soft, neither bleeding nor sensitive to touch, and the probe could be passed all around the mass. On nasal endoscopy, the anterior rhinoscopy findings were confirmed. The mass was seen arising from the right middle meatus with thick mucopurulent discharge, extending anteriorly till the anterior end of the inferior turbinate and posteriorly to the choana. Middle turbinate was pushed medially. Left-sided nasal mucosa was pale with mucoid discharge. Septum was deviated towards the left. Oral cavity examination showed multiple slough covered punched out ulcerations over a thickened, eroded uvula (Figure 1) and left side of soft palate with thick, post-nasal discharge. Gag reflex was present. Nasopharyngeal examination showed a mass arising from the upper surface soft palate extending into the choana with a thickened ulcerative mucosa of the nasopharyngeal surface of soft palate and onto the left lateral wall of the nasopharynx. Fossae of Rosenmuller on both sides were not involved. There was no external nasal or facial deformity or cervical lymphadenopathy.

Figure 1: Clinical picture showing the nasopharyngeal mass extending to the soft palate

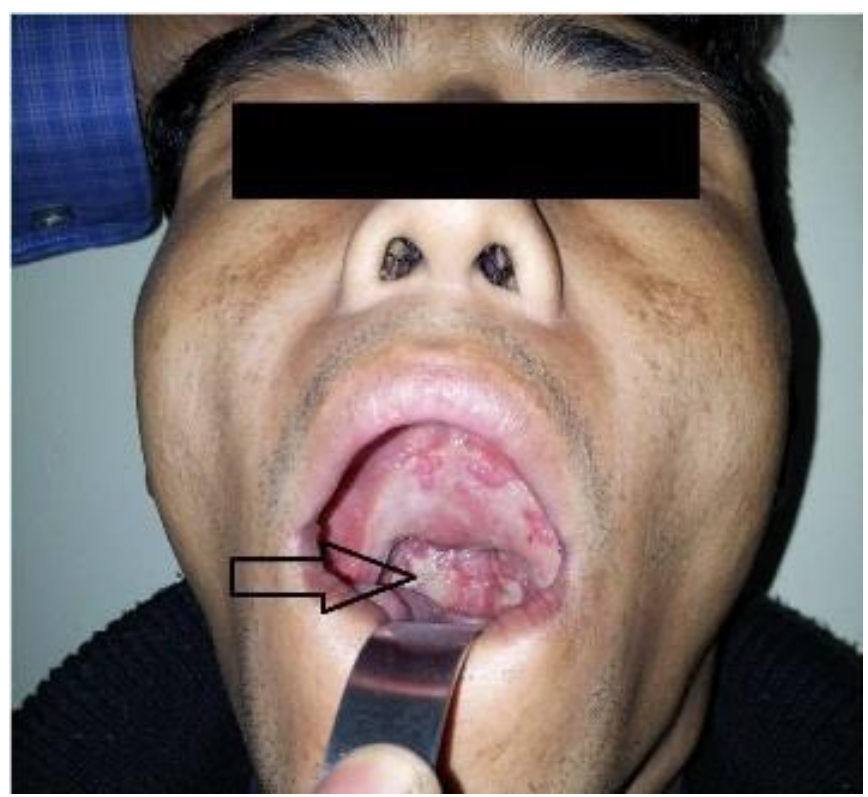

A contrast-enhanced computed tomography (CECT) (Figure

2 ) of the nose and paranasal sinuses with axial and coronal sections sinuses showed soft tissue density in the right maxillary (medial portion) and ethmoid sinuses and nasal cavity, suggestive of right sinonasal polyposis along with a soft tissue density in the nasopharynx towards the left side measuring about $19 \mathrm{~mm} \times 17 \mathrm{~mm}$. There was no significant contrast enhancement or any bony erosion. Nasal endoscopy with biopsy was done. Two separate samples were taken from the right cavity and the left nasopharynx for histopathological examination.

Figure 2: CECT image showing the right-sided nasal mass

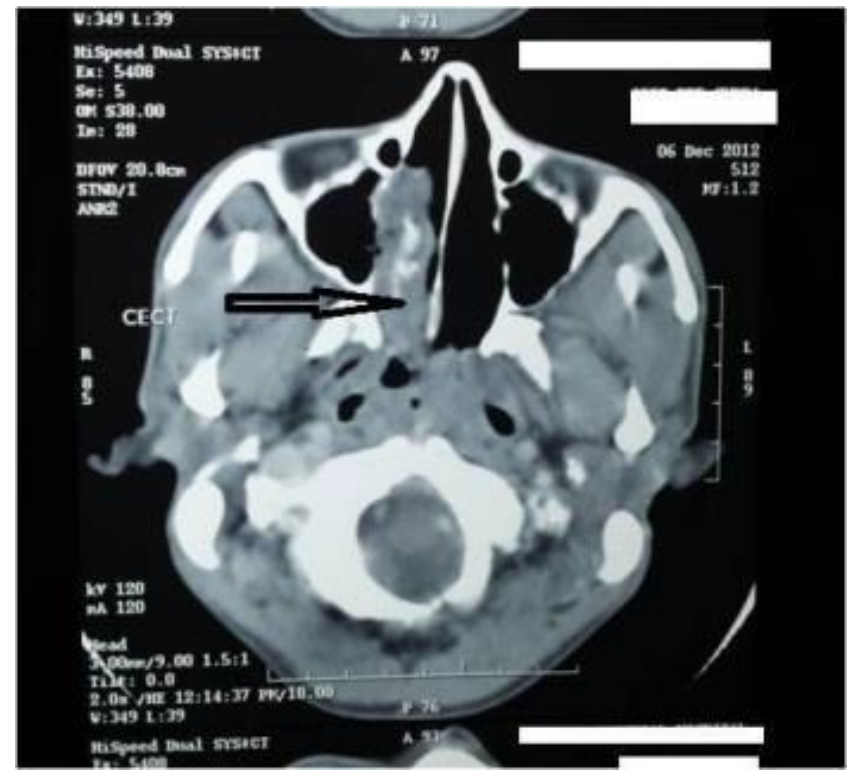

Histopathology of the nasopharyngeal mass revealed ulcerated tissue, densely populated by atypical lymphoid cells (Figure 3 ) with large areas of necrosis. The cells were small to medium sized with irregularly folded and angulated nuclei, dense chromatin, and inconspicuous nucleoli. Occasional large cells with clear cytoplasm were noted. Distortion and destruction of mucosal glands and vessels by these cells were seen. Immunohistochemistry for CD56 showed positive membranous staining in these cells (Figure 4). Histopathology of the right nasal mass showed features of inverted papilloma (Figure 5). The final diagnosis was NK/T-cell lymphoma with inverted papilloma.

The right nasal mass was debulked and the patient was started on chemotherapy with follow-up and has been referred outside the state for radiotherapy. No recurrence was noted at the last follow-up at 10 months. 
Figure 3: Histopathology showing sheets of atypical lymphoid cells with irregular angulated nuclei (H and $E$, 400X)

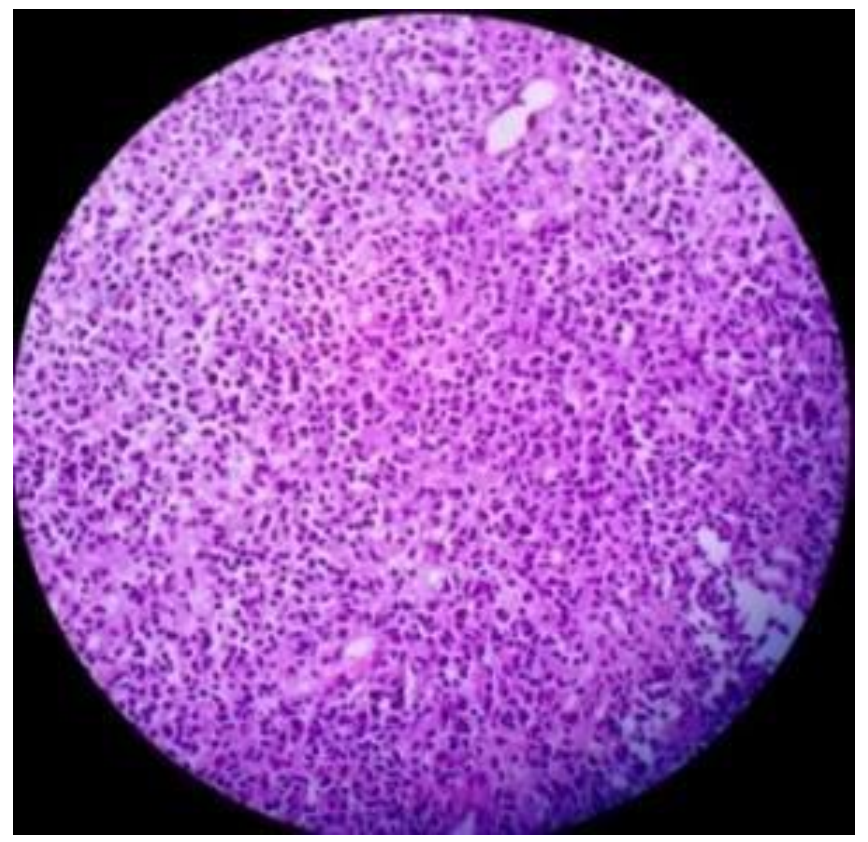

Figure 4: Immunohistochemistry showing CD 56 positivity in the neoplastic cells (400X)

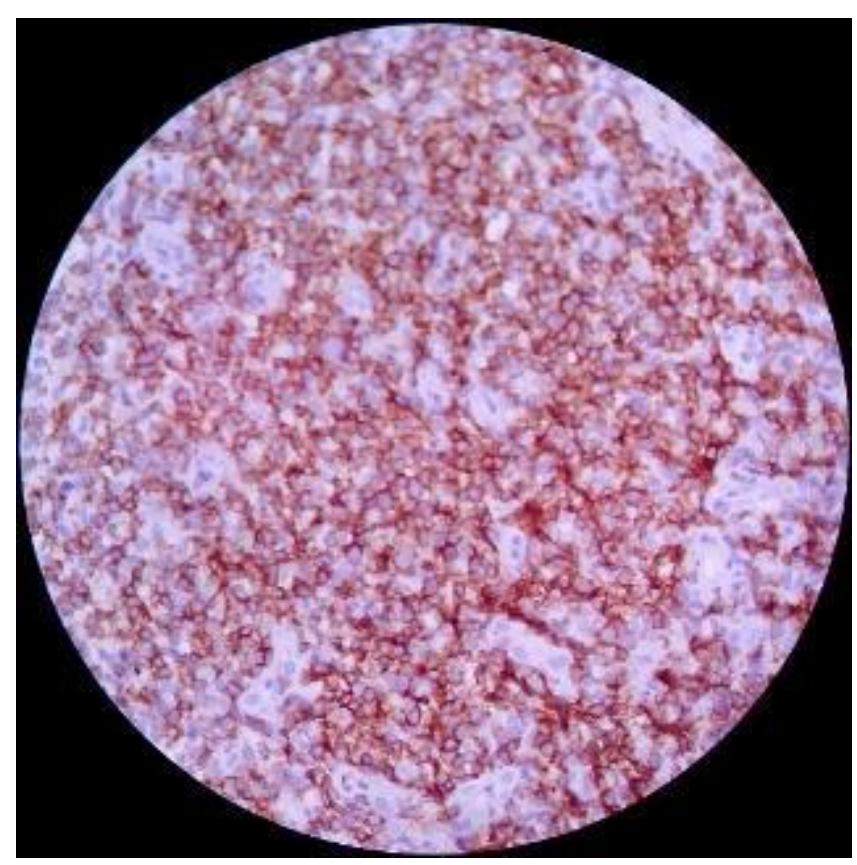

Figure 5: Histopathology of the associated inverted papilloma ( $H$ and $E, 100 X$ )

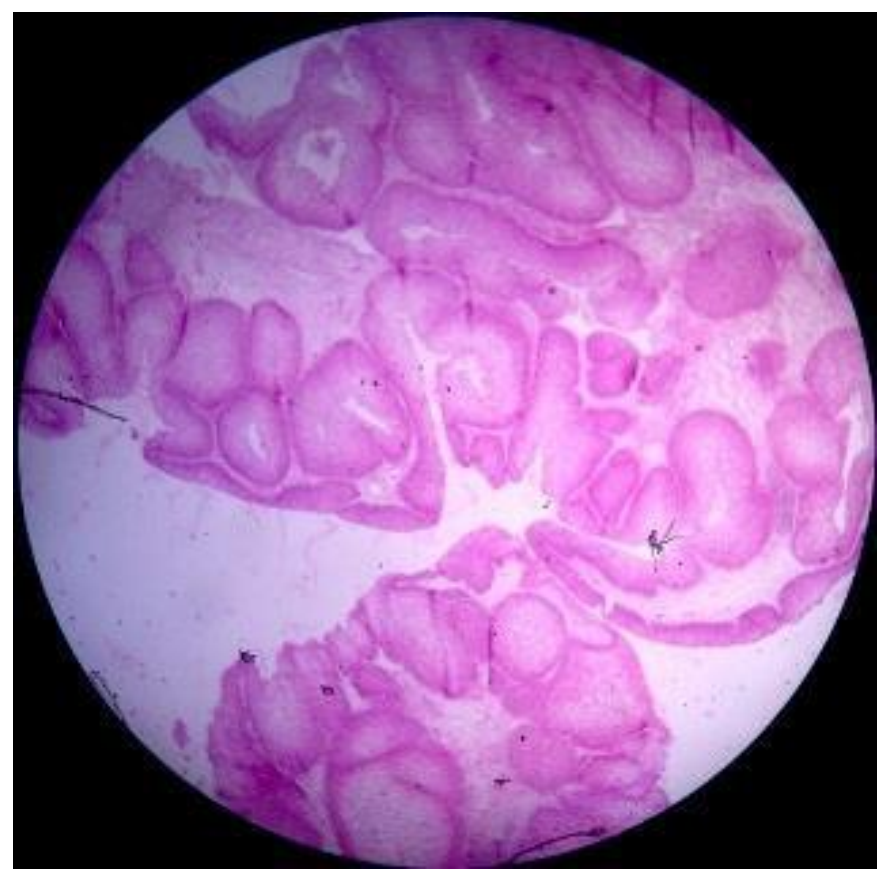

\section{Discussion}

Nasal-type NK/T-cell lymphoma is an uncommon neoplasm accounting for 2-8 per cent of non-Hodgkin lymphomas in Asia, where this entity shows its highest prevalence. In Europe and North America, nasal-type NK/T-cell lymphoma represents fewer than 2 per cent of non-Hodgkin lymphomas. ${ }^{1}$ Previously known as lethal midline granuloma, this neoplasm is said to be causally associated with Epstein Barr virus (EBV). ${ }^{1,2}$

It is characterised as a very aggressive disease, with local destroying activity, and refractoriness to treatment. The tumour bulk can be responsible, at presentation, for respiratory obstruction and/or periorbitary oedema. Infiltrating lesions show a destroying activity against soft and bony oropharyngeal and nasal structures, as was seen in our patient with uvular necrosis. It can spread to the central nervous system, cutis, and testis, and be responsible for hemophagocytosis in bone marrow. ${ }^{4}$ Tumour behaviour is aggressive with a reported median overall survival ranging from 13 to 38 months. ${ }^{1}$

On histopathology, an angiocentric/angiodestructive growth pattern with fibrinoid changes in the blood vessels with frequent coagulative necrosis as a consequence of vascular occlusion is observed. ${ }^{4}$ In most cases, the lymphoma is composed of neoplastic lymphocytes ranging from small cytologically bland cells, which may create a diagnostic dilemma, to large cells that are frankly malignant. Most cases show a morphologic spectrum with intermediatesized and large cells predominating with irregular nuclei, 
granular chromatin and mitotic figures. ${ }^{1,4}$ Neoplastic cells are usually admixed with an inflammatory infiltrate containing small lymphocytes, histiocytes, neutrophils, and eosinophils. ${ }^{1}$ When inadequate biopsies yield only inflammatory infiltrate, a false-negative diagnosis becomes inevitable. Hence, it is important to sample adequate representative areas of the lesion to avoid a false-negative diagnosis.

Neoplastic cells are surface CD3-, cytoplasmic $\mathrm{CD} 3 \varepsilon+$, CD56+, cytotoxic-molecule positive, Epstein-Barr virus positive, with germline T-cell receptor gene. ${ }^{1,2,4}$ The lesion on the right side in our patient showed all the above features of atypical small- to medium-sized lymphocytes with immunohistochemistry positive for $C D 56$, confirming our diagnosis of NK/T-cell lymphoma.

Inverted papillomas, by contrast, are uncommon, benign sinonasal tumours that are locally aggressive with a significant malignant potential. Nasal obstruction or perception of a nasal mass is the most common presenting symptom. Almost equally the papillomas may be endophytic (inverted) or exophytic (fungiform), and a few may have mixed patterns. ${ }^{5}$

The concurrent or sequential malignancies associated with inverted papilloma are mostly squamous cell carcinomas; the median rate of malignant association reported being approximately 9 per cent. ${ }^{6}$ Despite an extensive literature search, no case of simultaneous occurrence with T-cell lymphoma as seen in our case could be found. The presence of Epstein Barr virus had been reported in both NK/T-cell lymphomas ${ }^{1,2,4}$ and inverted papillomas, ${ }^{7}$ but subsequent studies have refuted any association between Inverted papilloma and Epstein Barr virus. ${ }^{8,9}$

For localised NK/T-cell lymphomas, radiotherapy together with chemotherapy is the standard approach. In disseminated disease, systemic chemotherapy remains the mainstay of treatment. ${ }^{2,4}$ Endoscopic surgical techniques and a more conservative approach is reserved for less widespread and more medially located inverted papillomas, while in tumours extending more peripherally from the lateral nasal wall or with concomitant malignancy a more aggressive approach to the papilloma, even an external, en bloc resection is preferred. ${ }^{6}$

In our patient, both the papilloma and the lymphoma were localised, and therefore a conservative approach was chosen. Though aggressive treatment of Schneiderian papillomas with concurrent squamous cell carcinoma is advocated, $^{6}$ no such guidelines were available for its treatment with other varieties of concurrent malignancies, causing a therapeutic dilemma. The patient is doing well on this treatment and there has been no evidence of any recurrence of either pathology on the six-month follow-up.

Another important factor to be considered is the delayed diagnosis of the diseases, as happened in this case, where the patient had symptoms of nasal discharge from one year before presentation to the specialist. Presentation to a family doctor with nasal congestion is not uncommon. Such patients are often treated for chronic sinusitis as symptoms include nasal stuffiness, post-nasal drip, facial fullness, and malaise for a period of 12 weeks or longer. The treatment comprises an adequate, preferably culture-directed antibiotic trial for three to four weeks and supportive treatment. Diagnostic work-up must include a diagnostic nasal endoscopy. CT scanning is the imaging standard and best done after antibiotic therapy. Serious underlying conditions, such as tumours and immunodeficiency states are always considered in the work-up. Benign as well as malignant tumours of the sinonasal cavities, nasopharynx, and skull base must be considered in the differential. ${ }^{10}$ This patient had a history of the said symptoms for more than a year. Any history of longstanding nasal obstruction, particularly lasting more than 12 weeks, even after an antibiotic trial of three weeks must be referred to an otolaryngologist. The blood-tinged nasal discharge necessitated immediate referral as many benign and malignant tumours may cause epistaxis. ${ }^{10}$ Ideally, the patient should have been referred to a specialist within three weeks of treatment, as his condition did not resolve. A nasal endoscopy and CT scan would have detected both the tumours quickly and treatment could have been instituted immediately. If this had not been done, the blood-tinged nasal discharge should alert the healthcare provider. Thus, the patient had an avoidable diagnostic delay of at least two months, if not 11 months from the initial presenting complaint.

Family doctors should refer patients to specialist care if cases of nasal congestion, especially those with bloodtinged rhinorrhea, do not completely resolve within 12 weeks after onset In cases similar to that presented here. General practitioners must also ensure that once patients have been treated for the more aggressive condition of the two (NK/T-cell lymphoma) that surgery for the second pathology (inverted papilloma) is considered. Inverted papillomas have a tendency to be locally malignant or undergo malignant transformation to squamous cell carcinoma, which can be prevented by their complete removal. $^{5,6}$ 


\section{Conclusion}

Inverted papillomas are known to be associated with hidden or apparent squamous cell carcinoma, not with nasal-type NK/T-cell lymphomas. Still, the presence of one type of tumour does not obviate the occurrence of another entity. This may pose diagnostic and therapeutic challenges, as was present in our case. A thorough and detailed examination of any sinonasal mass supplemented with histopathology and immunohistochemistry is vital for appropriate planning of treatment. Further, the association of Epstein Barr virus with inverted papillomas needs to be re-investigated, to support the likely co-existence of both the entities.

\section{References}

1. Schwartz EJ, Molina-Kirsch H, Zhao S, Marinelli RJ, Warnke RA, Natkunam Y. Immunohistochemical characterization of nasal-type extranodal NK/T-cell lymphoma using a tissue microarray: an analysis of 84 cases. Am J Clin Pathol. 2008;130(3):343-51.

2. Tse E, Kwong Y-L. How I treat NK/T-cell lymphomas. Blood. 2013;121(25):4997-5005.

3. Li YX, Liu QF, Fang $H$, Qi SN, Wang $H$, Wang $W H$, Song YW, Lu J, Jin J, Wang SL, Liu YP, Lu N, Liu XF, Yu ZH. Variable clinical presentations of nasal and Waldeyer ring natural killer/T-cell lymphoma. Clin Cancer Res 2009;15(8):2905-12.

4. Pagano L, Gallamini A, Trapè G, Fianchi L, Mattei D, Todeschini G, Spadea A, Cinieri S, Iannitto E, Martelli M, Nosari A, Bona ED, Tosti ME, Petti MC, Falcucci P, Montanaro M, Pulsoni A, Larocca LM, Leone G; Intergruppo Italiano Linfomi. Intergruppo Italiano Linfomi. NK/T-cell lymphomas 'nasal type': an Italian multicentric retrospective survey. Ann Oncol 2006;17(5):794-800.

5. Christensen WN, Smith RRL. Schneiderian papillomas: A clinicopathologic study of 67 cases. Hum Pathol 1986;17(4):393-400.

6. Krouse JH. Development of a Staging System for Inverted Papilloma. Laryngoscope 2000;110:965-68.

7. Macdonald MR, Le KT, Freeman J, Hui MF, Cheung RK, Dosch HM. A majority of inverted sinonasal papillomas carries Epstein Barr virus genomes. Cancer 1995;75:2307-12.

8. Gaffey MJ, Frierson HF, Weiss LM, Barber CM, Baber $\mathrm{GB}$, Stoler MH. Human papillomavirus and Epstein-Barr virus in sinonasal Schneiderian papillomas. An in situ hybridization and polymerase chain reaction study. Am J Clin Pathol. 1996;106(4):475-82.

9. Dunn ST, Clark GD, Cannon TC, Min KW. Survey of sinonasal inverted papillomata for Epstein-Barr virus. Head Neck 1997;19(2):98-106.
10. Brook I, Hinthon DR. Chronic Sinusitis. [updated 2014 April 7; cited 2014 July 11]. Available from: http://emedicine.medscape.com/article/232791

\section{PEER REVIEW}

Not commissioned. Externally peer reviewed.

\section{CONFLICTS OF INTEREST}

The authors declare that they have no competing interests.

\section{ETHICS COMMITTEE APPROVAL}

Not taken, as it is a single case report.

\section{PATIENT CONSENT}

The authors, Deb Roy A, Tuli IP, and Joshi D declare that:

1. They have obtained written, informed consent for the publication of the details relating to the patient(s) in this report.

2. All possible steps have been taken to safeguard the identity of the patient(s).

3. This submission is compliant with the requirements of local research ethics committees. 